\title{
Construction of new solitary wave solutions of generalized Zakharov-Kuznetsov-Benjamin- Bona-Mahony and simplified modified form of Camassa-Holm equations
}

https://doi.org/10.1515/phys-2018-0111

Received July 6, 2018; accepted September 25, 2018

\begin{abstract}
In this research work, for the first time we introduced and described the new method, which is modified extended auxiliary equation mapping method. We investigated the new exact traveling and families of solitary wave solutions of two well-known nonlinear evaluation equations, which are generalized Zakharov-KuznetsovBenjamin-Bona-Mahony and simplified modified forms of Camassa-Holm equations. We used a new technique and we successfully obtained the new families of solitary wave solutions. As a result, these new solutions are obtained in the form of elliptic functions, trigonometric functions, kink and antikink solitons, bright and dark solitons, periodic solitary wave and traveling wave solutions. These new solutions show the power and fruitfulness of this new method. We can solve other nonlinear partial differential equations with the use of this method.
\end{abstract}

Keywords: Modified extended auxiliary equation mapping method; GZK-BBM equation; modified form of $\mathrm{CH}$ equation; exact and Traveling wave solutions; solitary wave solutions

PACS: 02.30.Jr, 04.30.Nk, 05.45.Yv

\section{Introduction}

A few years ago many authors found the different types of exact traveling and solitary wave solutions

\footnotetext{
Dianchen Lu, Mujahid Iqbal: Faculty of Science, Jiangsu University, Zhenjiang, Jiangsu 212013, P. R. China

^Corresponding Author: Aly R. Seadawy:

Mathematics Department, Faculty of Science, Taibah University, Al-Madinah Al-Munawarah, Saudi Arabia

Mathematics Department, Faculty of Science, Beni-Suef University, Egypt, E-mail: Aly742001@yahoo.com
}

of both nonlinear models GZK-BBM and GCH-equations. These both equations are well-known nonlinear evaluation equations and play important role in many scientific fields. The GZK-BBM equation used in the studies of acoustic waves, acoustic-gravity waves, surface waves with long wavelength, hydromagnetic waves, these all mentioned waves have source harmonic crystals, compressible fluids, cold plasma and liquids, respectively. The GCH equation play important role in shallow water waves. In 2005 Wazwaz [1] studied the GZK-BBM equation for the first time and found some complex solutions, kink type solutions, periodic wave solutions and solitons solutions with the help of the sine-cosine method. Wazwaz [2] found the two types of compactons and solitary patterns wave solutions of ZK-BBM equation by applying the extended tanh method. Abdou [3] found the set of exact solutions of ZK-BBM equation with the help of extended F-Expansion method. Mahmoudi et al. [4] investigated the periodic solitary wave solutions of ZK-BBM equation by applying the exp-function method. Wang and Tang [5] studied the existence property of smoothness of traveling wave solutions of ZK-BBM equation by apply the bifurcation theory of planner. Song and Yang [6] with the help of bifurcation technique found the traveling wave, solitary wave and kink type solutions of ZK-BBM equation.

Camassa and Holm [7] derived a Camassa-Holm equation (CH-equation) by using the Hamiltonian methods, which is a completely integrable dispersive water waves equation by holding two terms, which are neglected in the limit of shallow water waves, having small amplitude. After that many authors started to investigate the different types of travelling solitary wave solutions of $\mathrm{CH}$ equation by using various methods. Cooper and Shepard [8] found the solitary wave solutions of GCH-equation by using the variational function. Liu et al. [9] improved? $\mathrm{CH}$ equation and found traveling wave solutions. Zhang and Bi [10] studied the bifurcation technique of $\mathrm{CH}$-equation. Liu and Tang [11] investigated the bifurcation phenomena 
and found the periodic solutions of GCH-equation with the help of integrated scheme. Deng et al. [12] found the compacton, kink and anti-kink, periodic solitary wave and solitons solutions of GCH-Degaspersi-Procesi-equation. Kalla and Klein [13] found the multidimensional theta functions independent derivation solutions of GCH equation with the help of technique that is related to Fay's identity.

Recently, Liu and Song [14] found the smooth periodic and blow-up periodic solutions of GZK-BBM equation by applying the bifurcation method. Khadijo Adem and Masood Khalique [15] investigated the traveling waves solutions and conservation laws of GZK-BBM equation with the help of $\left(G^{\prime} / G\right)$ - expansion method. Harun-Or-Roshid et al. [16] found the families of solitary waves solutions of GZK-BBM and RLW equations by using the modified simple equation method. Seadawy et al. [17] found the families of exact travelling and solitary wave solutions of GZK-BBM equation with the help of $\exp (-\varphi(\xi))$-expansion method. Many other authors have investigated the travelling solitary wave solutions of GZK-BBM equation and GCH-equation see Ref. [18-20, 26-30].

The nonlinear system of partial differential equations is very useful to study the physical nature in many different scientific fields, such as engineering, physics, geophysics, optics, chemistry, biology, material science, computer science, mechanics, electricity, ultrasound, thermodynamics and so on. The solitary and travelling wave solutions of NPDEs have many applications to understanding the process and physical phenomena in many areas of applied science. In the last five decades a lot of new methods have been developed by many groups of mathematicianas and engineers to investigate the (NPDEs). For example some important methods such as, exp-function method; modified Extended tanh-expansion method; modified simple equation method; homotopy perturbation method; novel $(G / G)$ - expansion method; extended modified direct algebraic method; generalized kudryashov method; modified extended Kudryashov method; $\exp (-\varphi(\xi))$-expansion method; extended Jacobian method; extended trial equation method and so on [31, 32, 35-50].

The main aim of this research is to investigate the exact traveling and solitary wave solutions of GZK-BBM and simplified modified form of $\mathrm{CH}$-equations. These new solutions are obtained with the help of new method, which is modified extended auxiliary equation mapping method. The arrangement of this article is organized as fellows. Description of the modified extended auxiliary equation mapping method is given in Section 2. Section 3 deals with the investigation of the solitary wave solutions of GZKBBM-equation and simplified modified CH-equation by us- ing the described method. Finally, the conclusion are presented in Section 4.

\section{Modified extended auxiliary equation mapping method}

Consider the general form of (2+1)-dimensional NPDEs as

$$
F\left(U, U_{t}, U_{x}, U_{y}, U_{x t}, \ldots\right)=0,
$$

here $F$ denotes the polynomial function of $U(x, y, t)$ and its all derivatives which contained highest order nonlinear terms and highest order partial derivatives. Here we explain the important steps of the new method as:

Step1. We apply the traveling wave transformations as

$$
U(x, y, t)=U(\xi), \xi=l x+m y+\omega t,
$$

where 1 and $\mathrm{m}$ are the wave numbers and $\omega$ is the frequency of the wave. We obtained the ODE of Eq.(1) as

$$
P\left(U, U^{\prime}, U^{\prime \prime}, U^{\prime \prime}, \ldots\right)=0,
$$

here $P$ is the polynomial function in $U(\xi)$ and its derivatives.

Step2. We consider the general solution of Eq.(2), in the following form

$$
\begin{aligned}
& U(\xi)=\sum_{i=0}^{n} a_{i} \Psi(x)^{i}+\sum_{i=-1}^{-n} b_{-i} \Psi(x)^{i}+\sum_{i=2}^{n} c_{2} \Psi(x)^{i-2} \Psi^{\prime}(x) \\
& +\sum_{i=1}^{n} d_{i}\left(\frac{\Psi^{\prime}(x)}{\Psi(x)}\right)^{i}
\end{aligned}
$$

where $a_{0}, a_{1}, \ldots a_{n}, b_{1}, b_{2}, \ldots b_{n}, c_{2}, c_{3}, \ldots c_{n}, d_{1}, d_{2}, \ldots d_{n}$ are constants parameter to be find later, the values of $\Psi(\xi)$ and its derivative $\Psi^{\prime}(\xi)$ satisfy to the given auxiliary equation

$$
\begin{aligned}
\Psi^{\prime}(\xi)= & \sqrt{\beta_{1} \Psi^{2}(\xi)+\beta_{2} \Psi^{3}(\xi)+\beta_{3} \Psi^{4}(\xi)} \\
\Psi^{\prime \prime}(\xi)= & \beta_{1} \Psi(\xi)+\frac{3}{2} \beta_{2} \Psi^{2}(\xi)+2 \beta_{3} \Psi^{3}(\xi) \\
\Psi^{\prime \prime \prime}(\xi)= & \left(\beta_{1}+3 \beta_{2} \Psi(\xi)+6 \beta_{3} \Psi^{2}(\xi)\right) \Psi^{\prime}(\xi) \\
\Psi^{\prime \prime \prime \prime}(\xi)= & \frac{1}{2} \Psi(\xi)\left(2 \beta_{1}^{2}+15 \beta_{1} \beta_{2} \Psi(\xi)+5\left(3 \beta_{2}^{2}+8 \beta_{1} \beta_{3}\right) \Psi^{2}(\xi)\right. \\
& \left.+60 \beta_{2} \beta_{3} \Psi^{3}(\xi)+48 \beta_{3}^{2} \Psi^{4}(\xi)\right)
\end{aligned}
$$

Where $\beta_{i}^{\prime} s$ are real constants, which determine later such that $\beta_{n} \neq 0$.

Step3. Balance of the highest order nonlinear terms and highest order partial derivatives in Eq. (3) is found to be 
the positive integer $n$ of Eq. (3).

Step4. Substituting Eq. (5) into Eq. (4) and combining each coefficients of $\Psi^{i}(\xi)(i=1,2,3, \ldots n)$, then making a every coefficient equal to zero and obtaining a families of algebraic equations, solving this system of equations with the help of Mathematica, the constants $a_{0}, a_{1}, \ldots a_{n}, b_{1}, b_{2}, \ldots b_{n}, c_{2}, c_{3}, \ldots c_{n}, d_{1}, d_{2}, \ldots d_{n} \quad$ can be determined.

Step5. Substituting the values of obtained constants and $\Psi(\xi)$ into Eq. (3), then required solutions of Eq. (1) are obtained.

\section{Application of the proposed method}

Now we applying the modified extended auxiliary equation mapping method to investigate the families of new solitary wave solutions for the $(2+1)$-dimensional GZK-BBM-equation and simplified modified form of $\mathrm{CH}$ equation.

\subsection{Generalized Zakharov-Kuznetsov- Benjamin-Bona-Mahony equation}

We consider a (2+1)-dim GZK-BBM-equation as

$$
U_{t}+U_{x}+\alpha\left(U^{3}\right)_{x}+\gamma\left(U_{\mathrm{xt}}+U_{\mathrm{yy}}\right)_{x}=0,
$$

where $\gamma$ and $\alpha$ are non zero constants. Consider the traveling wave transformation $U(x, y, t)=U(\xi), \xi=l x+m y+\omega t$, by this transformation we obtained ordinary differential equation of Eq.(6) as

$$
\omega U^{\prime}+1 U^{\prime}+3 \alpha \mathrm{l}^{2} U^{\prime}+\gamma m^{3} U^{\prime \prime \prime}+\gamma \mathrm{l}^{2} \omega U^{\prime \prime \prime}=0,
$$

we integrate the Eq.(7) once time according to $\xi$ and integration constant equal to zero, then we obtained as

$$
\omega U+1 U+\alpha l U^{3}+\gamma l^{2} \omega U^{\prime \prime}+\gamma m^{3} U^{\prime \prime}=0,
$$

Balance the highest order nonlinear term and highest order partial derivative in Eq.(8) obtained the value of $n=$ 1. The general solution of Eq.(8) takes form of

$$
U(\xi)=a_{0}+a_{1} \Psi(\xi)+\frac{b_{1}}{\Psi(\xi)}+d_{1} \frac{\Psi^{\prime}(\xi)}{\Psi(\xi)}
$$

Substituting Eq. (9) into Eq. (8) and combining each coefficients of $\Psi^{\prime}{ }^{j}(\xi) \Psi^{i}(\xi)(j=0,1 ; i=1,2,3, \ldots n)$, then making a every coefficient equal to zero and obtaining a set of algebraic equations. We solve this system of equations with the aid of Mathematica. The parameters $a_{0}, a_{1}, b_{1}, d_{1}$ can be determined as

\section{Case-I}

$$
\begin{gathered}
a_{0}=0, a_{1}=a_{1}, b_{1}=0, d_{1}= \pm \frac{\sqrt{-l-\omega}}{\sqrt{\alpha} \sqrt{\beta_{1}} \sqrt{l}}, \beta_{3}= \pm \frac{\alpha a_{1}^{2} \beta_{1} l}{l+\omega}, \\
m= \pm \frac{(-1)^{2 / 3} \sqrt[3]{\beta_{1}(-\gamma) l^{2} \omega+2 l+2 \omega}}{\sqrt[3]{\beta_{1}} \sqrt[3]{\gamma}} .
\end{gathered}
$$

Substituting the Eq.(10), only for the positive value of $d_{1}$ in Eq. (9), then solitary wave solutions of Eq. (6) are obtained in simplified forms as:

$$
\begin{aligned}
U_{1}(x, y, t)=-\frac{\epsilon \sqrt{-l-\omega} \operatorname{csch}\left[\frac{1}{2} \sqrt{\beta_{1}}\left(l x+m y+\omega t+\xi_{0}\right)\right]^{2}}{\sqrt{\alpha} \sqrt{l}\left(2 \epsilon \operatorname{coth}\left[\frac{1}{2} \sqrt{\beta_{1}}\left(l x+m y+\omega t+\xi_{0}\right)\right]+2\right)} \\
-\frac{a_{1} \beta_{1}\left(1+\epsilon \operatorname{coth}\left[\frac{1}{2} \sqrt{\beta_{1}}\left(l x+m y+\omega t+\xi_{0}\right)\right]\right)}{\beta_{2}}
\end{aligned}
$$

$$
\begin{aligned}
U_{2}(x, y, t)= & \left(2 \epsilon \sqrt{-l-\omega}\left(\eta \cosh \left[\sqrt{\beta_{1}}\left(l x+m y+\omega t+\xi_{0}\right)\right]+1\right)\right. \\
& -\sqrt{\alpha} \sqrt{l}\left(\eta+\cosh \left[\sqrt{\beta_{1}}\left(l x+m y+\omega t+\xi_{0}\right)\right]\right. \\
& \left.\left.+\epsilon \sinh \left[\sqrt{\beta_{1}}\left(l x+m y+\omega t+\xi_{0}\right)\right]\right)^{2} a_{1} \sqrt{\frac{\beta_{1}}{\beta_{3}}}\right) / \\
& \left(2 \sqrt{\alpha} \sqrt{l}\left(\eta+\cosh \left[\sqrt{\beta_{1}}\left(l x+m y+\omega t+\xi_{0}\right)\right]\right)\right. \\
& \eta+\cosh \left[\sqrt{\beta_{1}}\left(l x+m y+\omega t+\xi_{0}\right)\right] \\
& \left.+\epsilon \sinh \left[\sqrt{\beta_{1}}\left(l x+m y+\omega t+\xi_{0}\right)\right]\right)
\end{aligned}
$$

$$
\begin{aligned}
& U_{3}(x, y, t)=\epsilon \sqrt{-l-\omega}\left(\eta \sqrt{p^{2}+1} \cosh \left[\sqrt{\beta_{1}}\left(l x+m y+\omega t+\xi_{0}\right)\right]\right. \\
& \left.-p \sinh \left[\sqrt{\beta_{1}}\left(l x+m y+\omega t+\xi_{0}\right)\right]+1\right) / \\
& \left(\sqrt { \alpha } \sqrt { l } \left(\cosh \left[\sqrt{\beta_{1}}\left(l x+m y+\omega t+\xi_{0}\right)\right]\right.\right. \\
& \left.+\eta \sqrt{p^{2}+1}\right)\left(\cosh \left[\sqrt{\beta_{1}}\left(l x+m y+\omega t+\xi_{0}\right)\right]\right. \\
& \left.\left.+\eta \sqrt{p^{2}+1}+\epsilon\left(\sinh \left[\sqrt{\beta_{1}}\left(l x+m y+\omega t+\xi_{0}\right)\right]+p\right)\right)\right) \\
& +a_{1}\left(-1-\frac{\epsilon\left(\sinh \left[\sqrt{\beta_{1}}\left(l x+m y+\omega t+\xi_{0}\right)\right]+p\right)}{\cosh \left[\sqrt{\beta_{1}}\left(l x+m y+\omega t+\xi_{0}\right)\right]+\eta \sqrt{p^{2}+1}}\right)
\end{aligned}
$$




\section{Case-II}

$$
a_{0}= \pm \frac{\sqrt{-l-\omega}}{\sqrt{\alpha} \sqrt{l}}, a_{1}=a_{1}, b_{1}=d_{1}=0, m= \pm \frac{\sqrt[3]{2(l+\omega)-\beta_{1} \gamma l^{2} \omega}}{\sqrt[3]{\beta_{1}} \sqrt[3]{\gamma}}, \beta_{2}= \pm \frac{\sqrt{\alpha} a_{1} \beta_{1} \sqrt{l}}{\sqrt{-l-\omega}}, \beta_{3}=-\frac{\alpha a_{1}^{2} \beta_{1} l}{4(l+\omega)} .
$$

Substituting Eq.(14), only the positive value of $a_{0}$ into Eq.(9), the solutions of Eq.(6) are given as:

$$
\begin{gathered}
U_{4}(x, y, t)=\frac{\sqrt{-l-\omega}}{\sqrt{\alpha} \sqrt{l}}-\frac{a_{1} \beta_{1}\left(1+\epsilon \operatorname{coth}\left[\frac{1}{2} \sqrt{\beta_{1}}\left(l x+m y+\omega t+\xi_{0}\right)\right]\right)}{\beta_{2}} \\
U_{5}(x, y, t)=\frac{\sqrt{-l-\omega}}{\sqrt{\alpha} \sqrt{l}}-\frac{1}{2} a_{1} \sqrt{\frac{\beta_{1}}{\beta_{3}}}\left(1+\frac{\epsilon \sinh \left[\sqrt{\beta_{1}}\left(l x+m y+\omega t+\xi_{0}\right)\right]}{\eta+\cosh \left[\sqrt{\beta_{1}}\left(l x+m y+\omega t+\xi_{0}\right)\right]}\right) \\
U_{6}(x, y, t)=\frac{\sqrt{-l-\omega}}{\sqrt{\alpha} \sqrt{l}}+a_{1}\left(-1-\frac{\epsilon\left(\sinh \left[\sqrt{\beta_{1}}\left(l x+m y+\omega t+\xi_{0}\right)\right]+p\right)}{\cosh \left[\sqrt{\beta_{1}}\left(l x+m y+\omega t+\xi_{0}\right)\right]+\eta \sqrt{p^{2}+1}}\right)
\end{gathered}
$$

\section{Case-III}

$$
a_{0}=a_{1}=b_{1}=0, d_{1}= \pm \frac{\sqrt{-l-\omega}}{\sqrt{\alpha} \sqrt{\beta_{1}} \sqrt{l}}, m= \pm \frac{(-1)^{2 / 3} \sqrt[3]{2(l+\omega)-\beta_{1} \gamma l^{2} \omega}}{\sqrt[3]{\beta_{1} \sqrt[3]{\gamma}}} .
$$

Substituting Eq.(18) into Eq.(9), then the solutions of Eq.(6) can be obtained as:

$$
\begin{aligned}
& U_{7}(x, y, t)=\frac{\epsilon \sqrt{-l-\omega} \operatorname{csch}\left[\frac{1}{2} \sqrt{\beta_{1}}\left(l x+m y+\omega t+\xi_{0}\right)\right]^{2}}{\sqrt{\alpha} \sqrt{l}\left(2 \epsilon \operatorname{coth}\left[\frac{1}{2} \sqrt{\beta_{1}}\left(l x+m y+\omega t+\xi_{0}\right)\right]+2\right)} \\
& U_{8}(x, y, t)=-\epsilon \sqrt{-l-\omega}\left(\eta \cosh \left[\sqrt{\beta_{1}}\left(l x+m y+\omega t+\xi_{0}\right)\right]+1\right) /\left(\sqrt{\alpha} \sqrt{l}\left(\eta+\cosh \left[\sqrt{\beta_{1}}\left(l x+m y+\omega t+\xi_{0}\right)\right]\right)\right. \\
& \left.\eta+\cosh \left[\sqrt{\beta_{1}}\left(l x+m y+\omega t+\xi_{0}\right)\right]+\epsilon \sinh \left[\sqrt{\beta_{1}}\left(l x+m y+\omega t+\xi_{0}\right)\right]\right) \\
& U_{9}(x, y, t)=\epsilon \sqrt{-l-\omega}\left(\eta\left(-\sqrt{p^{2}+1}\right) \cosh \left[\sqrt{\beta_{1}}\left(l x+m y+\omega t+\xi_{0}\right)\right]+p \sinh \left[\sqrt{\beta_{1}}\left(l x+m y+\omega t+\xi_{0}\right)\right]-1\right) / \\
& \left(\sqrt { \alpha } \sqrt { l } ( \operatorname { c o s h } [ \sqrt { \beta _ { 1 } } ( l x + m y + \omega t + \xi _ { 0 } ) ] + \eta \sqrt { p ^ { 2 } + 1 } ) \left(\cosh \left[\sqrt{\beta_{1}}\left(l x+m y+\omega t+\xi_{0}\right)\right]+\eta \sqrt{p^{2}+1}\right.\right. \\
& \left.\left.+\epsilon\left(\sinh \left[\sqrt{\beta_{1}}\left(l x+m y+\omega t+\xi_{0}\right)\right]+p\right)\right)\right)
\end{aligned}
$$




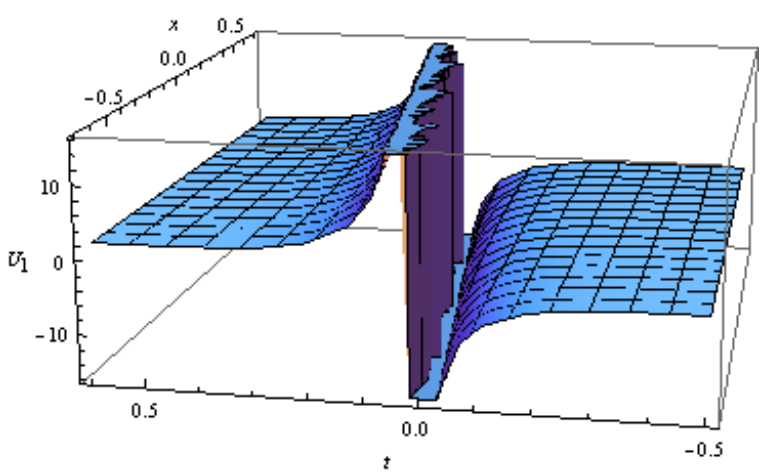

(a)

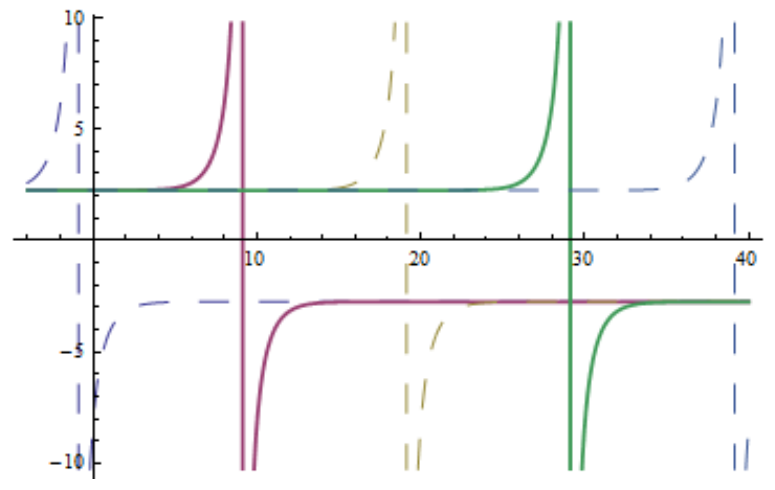

(b)

Figure 1: Solitary wave solution given in Eq.(11) when $a_{1}=0.5, \beta_{1}=2, \beta_{2}=4, \epsilon=10, \eta=-8, \xi_{0}=0.5, l=0.8, m=0.2, \omega=-8, y=1, a=$

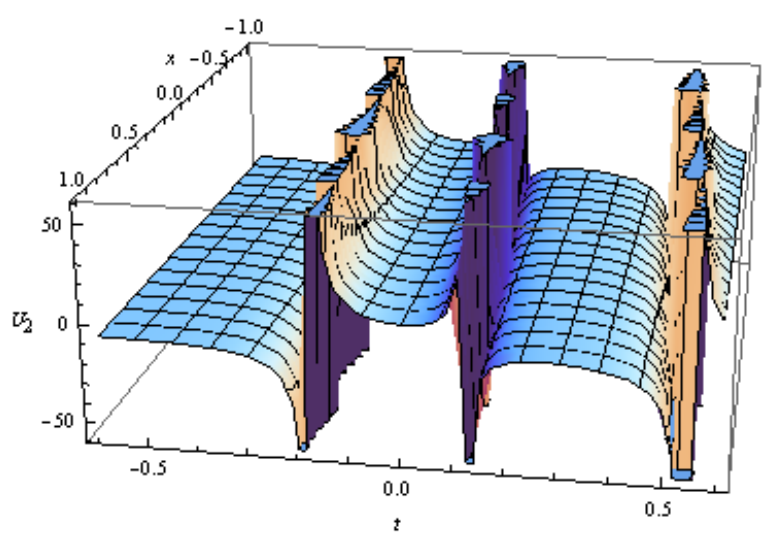

(a)

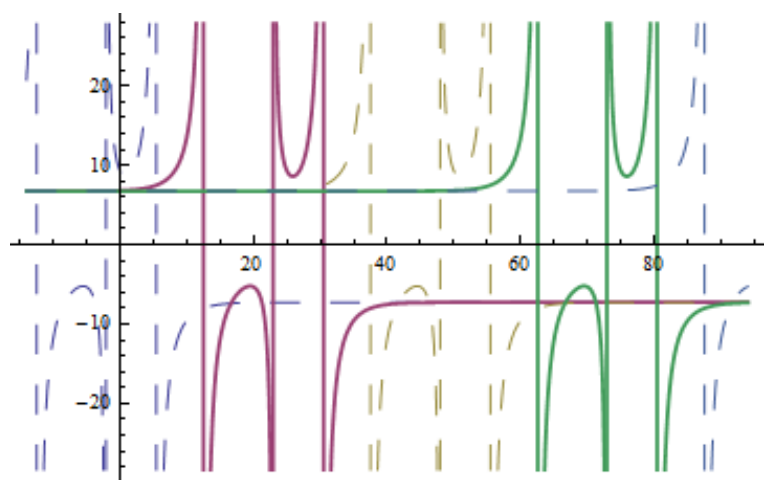

(b)

Figure 2: Solitary wave solution given in Eq.(12) when $a_{1}=0.5, \beta_{1}=4, \beta_{2}=8, \beta_{3}=4, \epsilon=28, \eta=-18, \xi_{0}=0.3, l=0.2, m=0.4, \omega=$ $-5, y=1, a=1$

\section{Case-IV}

$$
a_{0}= \pm \frac{\sqrt{-l-\omega}}{2 \sqrt{\alpha} \sqrt{l}}, a_{1}=b_{1}=0, d_{1}= \pm \frac{\sqrt{-l-\omega}}{2 \sqrt{\alpha} \sqrt{\beta_{1}} \sqrt{l}}
$$

Substituting Eq.(22), only the positive value of $a_{0}$ into Eq.(9), then the solutions of Eq.(6), can be given as:

$$
U_{10}(x, y, t)=\frac{\sqrt{-l-\omega}\left(\epsilon\left(\sinh \left[\sqrt{\beta_{1}}\left(l x+m y+\omega t+\xi_{0}\right)\right]-1\right) \operatorname{csch}\left[\frac{1}{2} \sqrt{\beta_{1}}\left(l x+m y+\omega t+\xi_{0}\right)\right]^{2}+2\right)}{4 \sqrt{\alpha} \sqrt{l}\left(\epsilon \operatorname{coth}\left[\frac{1}{2} \sqrt{\beta_{1}}\left(l x+m y+\omega t+\xi_{0}\right)\right]+1\right)}
$$




$$
\begin{aligned}
U_{11}(x, y, t)= & \left(\sqrt { - l - \omega } \left(1+2 \epsilon+2 \eta^{2}+2 \eta(\epsilon+2) \cosh \left[\sqrt{\beta_{1}}\left(l x+m y+\omega t+\xi_{0}\right)\right]+\cosh \left[2 \sqrt{\beta_{1}}\left(l x+m y+\omega t+\xi_{0}\right)\right]+\right.\right. \\
& \left.\left.2 \epsilon \sinh \left[\sqrt{\beta_{1}}\left(l x+m y+\omega t+\xi_{0}\right)\right]\left(\eta+\cosh \left[\sqrt{\beta_{1}}\left(l x+m y+\omega t+\xi_{0}\right)\right]\right)\right)\right) / \\
& \left(4 \sqrt { \alpha } \sqrt { l } ( \eta + \operatorname { c o s h } [ \sqrt { \beta _ { 1 } } ( l x + m y + \omega t + \xi _ { 0 } ) ] ) \left(\eta+\cosh \left[\sqrt{\beta_{1}}\left(l x+m y+\omega t+\xi_{0}\right)\right]\right.\right. \\
& \left.\left.+\epsilon \sinh \left[\sqrt{\beta_{1}}\left(l x+m y+\omega t+\xi_{0}\right)\right]\right)\right)
\end{aligned}
$$

$U_{12}(x, y, t)=\frac{\sqrt{-l-\omega}\left(\frac{\epsilon\left(\eta \sqrt{p^{2}+1} \cosh \left[\sqrt{\beta_{1}}\left(l x+m y+\xi_{0}+t \omega\right)\right]-p \sinh \left[\sqrt{\beta_{1}}\left(l x+m y+\xi_{0}+t \omega\right)\right]+1\right)}{\left(\cosh \left[\sqrt{\beta_{1}}\left(l x+m y+\xi_{0}+t \omega\right)\right]+\eta \sqrt{p^{2}+1}\right)\left(\epsilon\left(\sinh \left[\sqrt{\beta_{1}}\left(l x+m y+\xi_{0}+t \omega\right)\right]+p\right)+\cosh \left[\sqrt{\beta_{1}}\left(l x+m y+\xi_{0}+t \omega\right)\right]+\eta \sqrt{p^{2}+1}\right)}+1\right)}{2 \sqrt{\alpha} \sqrt{l}}$

\section{Case-V}

$$
a_{0}=a_{0}, a_{1}=0, b_{1}=\frac{2 a_{0} \beta_{1}}{\beta_{2}}, d_{1}=0, m=\frac{\sqrt[3]{-1} \sqrt[3]{\omega}}{\sqrt[3]{\beta_{1}} \sqrt[3]{\gamma}}
$$

Substituting Eq.(26) into Eq.(9), then the solutions of Eq.(6) can be obtained as:

$$
\left.\begin{array}{c}
U_{13}(x, y, t)=a_{0}\left(1-\frac{2}{\epsilon \operatorname{coth}\left[\frac{1}{2} \sqrt{\beta_{1}}\left(l x+m y+\omega t+\xi_{0}\right)\right]+1}\right) \\
U_{14}(x, y, t)=a_{0}\left(1-\frac{4 \sqrt{\frac{\beta_{1}}{\beta_{3}}} \beta_{3}}{\left.\beta_{2}+\frac{\beta_{2} \epsilon \sinh \left[\sqrt{\beta_{1}}\left(l x+m y+\omega t+\xi_{0}\right)\right]}{\eta+\cosh \left[\sqrt{\beta_{1}}\left(l x+m y+\omega t+\xi_{0}\right)\right]}\right)}\right. \\
U_{15}(x, y, t)=a_{0}\left(1-\frac{2 \beta_{1}}{\beta_{2}\left(\frac{\epsilon\left(\sinh \left[\sqrt{\beta_{1}}\left(l x+m y+\omega t+\xi_{0}\right)\right]+p\right)}{\cosh \left[\sqrt{\beta_{1}}\left(l x+m y+\omega t+\xi_{0}\right)\right]+\eta \sqrt{p^{2}+1}}+1\right)}\right)
\end{array}\right)
$$

\section{Case-VI}

$$
\begin{gathered}
a_{0}= \pm \frac{\sqrt{6 \beta_{1} \gamma \omega^{2}+9}}{2 \sqrt{\alpha\left(4 \beta_{1}^{2} \gamma^{2} \omega^{4}-9\right)}}, a_{1}=b_{1}=0, d_{1}= \pm \frac{\sqrt{6 \beta_{1} \gamma \omega^{2}+9}}{2 \sqrt{\alpha \beta_{1}\left(4 \beta_{1}^{2} \gamma^{2} \omega^{4}-9\right)}}, \\
l=\frac{3}{2 \beta_{1} \gamma \omega}-\omega, m= \pm \frac{\sqrt[3]{-1} \sqrt[3]{3-4 \beta_{1} \gamma \omega^{2}\left(\beta_{1} \gamma \omega^{2}-3\right)}}{2^{2 / 3} \beta_{1}^{2 / 3} \gamma^{2 / 3} \sqrt[3]{\omega}} .
\end{gathered}
$$


Substituting Eq.(30), only positive value of $a_{0}$ and $d_{1}$ into Eq.(9), the solitary wave solutions of Eq.(6) can be obtained in the simplified form as:

$$
U_{16}(x, y, t)=\frac{1}{4} \sqrt{6 \beta_{1} \gamma \omega^{2}+9}\left(\frac{2}{\sqrt{\alpha\left(4 \beta_{1}^{2} \gamma^{2} \omega^{4}-9\right)}}-\frac{\sqrt{\beta_{1}} \epsilon \operatorname{csch}\left[\frac{1}{2} \sqrt{\beta_{1}}\left(l x+m y+\omega t+\xi_{0}\right)\right]^{2}}{\sqrt{\alpha \beta_{1}\left(4 \beta_{1}^{2} \gamma^{2} \omega^{4}-9\right)}\left(\epsilon \operatorname{coth}\left[\frac{1}{2} \sqrt{\beta_{1}}\left(l x+m y+\omega t+\xi_{0}\right)\right]+1\right)}\right)
$$

$$
\begin{aligned}
& \begin{aligned}
U_{17}(x, y, t)= & \frac{1}{2} \sqrt{6 \beta_{1} \gamma \omega^{2}+9}\left(\frac{1}{\sqrt{\alpha\left(4 \beta_{1}^{2} \gamma^{2} \omega^{4}-9\right)}}+\sqrt{\beta_{1}} \epsilon\left(\eta \cosh \left[\sqrt{\beta_{1}}\left(l x+m y+\omega t+\xi_{0}\right)\right]+1\right) /\right. \\
& \left(\eta+\cosh \left[\sqrt{\beta_{1}}\left(l x+m y+\omega t+\xi_{0}\right)\right]\left(\eta+\cosh \left[\sqrt{\beta_{1}}\left(l x+m y+\omega t+\xi_{0}\right)\right]\right.\right. \\
& \left.\left.\left.+\epsilon \sinh \left[\sqrt{\beta_{1}}\left(l x+m y+\omega t+\xi_{0}\right)\right]\right) \sqrt{\alpha \beta_{1}\left(4 \beta_{1}^{2} \gamma^{2} \omega^{4}-9\right)}\right)\right) \\
U_{18}(x, y, t)= & \sqrt{6 \beta_{1} \gamma \omega^{2}+9}\left(\frac{1}{\sqrt{\alpha\left(4 \beta_{1}^{2} \gamma^{2} \omega^{4}-9\right)}}+\left(\epsilon \left(\eta \sqrt{p^{2}+1} \cosh \left[\sqrt{\beta_{1}}\left(l x+m y+\omega t+\xi_{0}\right)\right]+1\right.\right.\right. \\
& \left.\left.-p \sinh \left[\sqrt{\beta_{1}}\left(l x+m y+\omega t+\xi_{0}\right)\right]\right) \sqrt{\beta_{1}}\right) /\left(\cosh \left[\sqrt{\beta_{1}}\left(l x+m y+\omega t+\xi_{0}\right)\right]+\eta \sqrt{p^{2}+1}\right. \\
& \left(\cosh \left[\sqrt{\beta_{1}}\left(l x+m y+\omega t+\xi_{0}\right)\right]+\eta \sqrt{p^{2}+1}+\epsilon\left(\sinh \left[\sqrt{\beta_{1}}\left(l x+m y+\omega t+\xi_{0}\right)\right]+p\right)\right) \\
& \left.\left.\sqrt{\alpha \beta_{1}\left(4 \beta_{1}^{2} \gamma^{2} \omega^{4}-9\right)}\right)\right)
\end{aligned}
\end{aligned}
$$

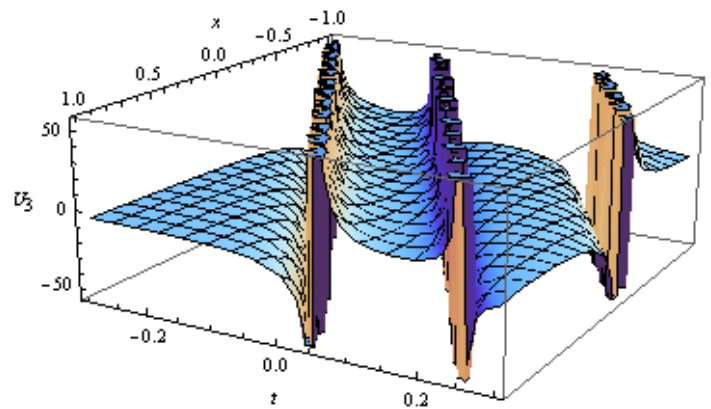

(a)

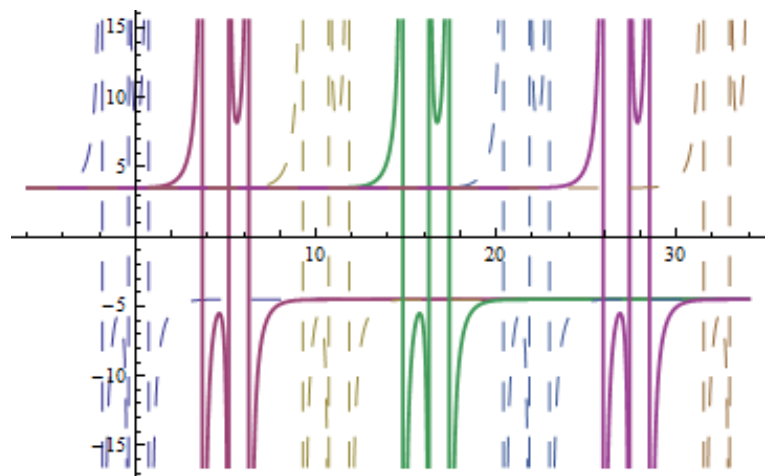

(b)

Figure 3: Solitary wave solution given in Eq.(13) when $a_{1}=0.5, \beta_{1}=4, \epsilon=8, \eta=-5, \xi_{0}=0.3, l=0.9, m=0.2, \omega=-5, y=1, a=0.4, p=$ 0.2 


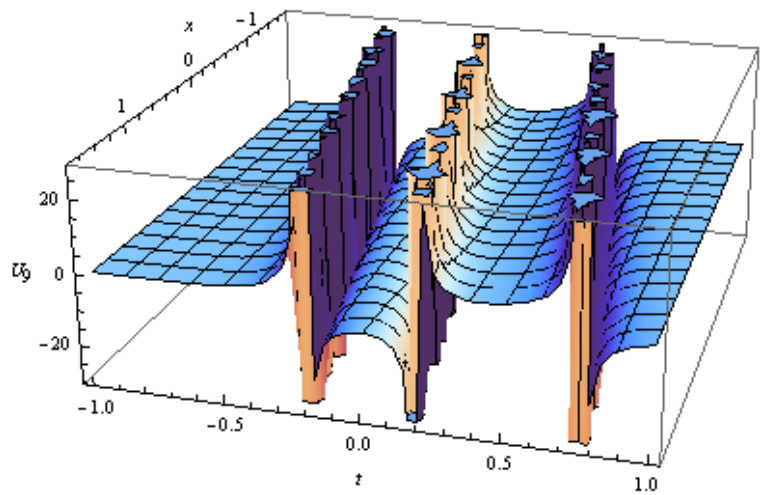

(a)

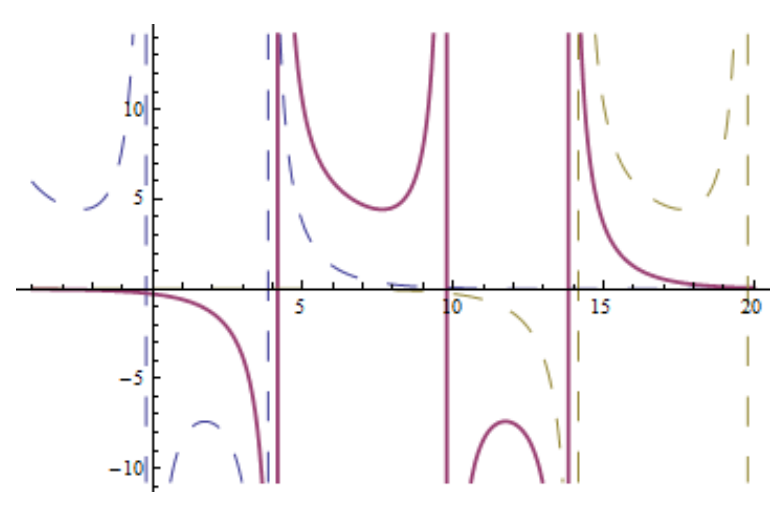

(b)

Figure 4: Solitary wave solution given in Eq.(21) when $a_{1}=0.4, \beta_{1}=2, \epsilon=18, \eta=-15, \xi_{0}=0.3, l=0.5, m=0.2, \omega=-5, y=1, a=$ $0.4, p=0.2$

\section{Case-VII}

$$
\begin{aligned}
& a_{0}= \pm \frac{\sqrt{1-\beta_{1}^{2} \gamma^{2} \omega^{4}}}{\sqrt{2} \sqrt{\alpha\left(\beta_{1} \gamma \omega^{2}-1\right)\left(\beta_{1} \gamma \omega^{2}+2\right)}}, a_{1}=b_{1}=0, \\
& d_{1}= \pm \frac{\sqrt{1-\beta_{1}^{2} \gamma^{2} \omega^{4}}}{\sqrt{2} \sqrt{\alpha \beta_{1}\left(\beta_{1} \gamma \omega^{2}-1\right)\left(\beta_{1} \gamma \omega^{2}+2\right)}}, l=\frac{2}{\beta_{1} \gamma \omega}+\omega, m=-(-1)^{2 / 3} \omega .
\end{aligned}
$$

Substituting Eq.(34), only the positive value of $a_{0}$ and $d_{1}$ into Eq.(9), then the solutions of Eq.(6) can be get in the simplified form as:

$$
U_{19}(x, y, t)=\frac{\sqrt{1-\beta_{1}^{2} \gamma^{2} \omega^{4}}\left(\frac{2}{\sqrt{\alpha\left(\beta_{1} \gamma \omega^{2}-1\right)\left(\beta_{1} \gamma \omega^{2}+2\right)}}-\frac{\sqrt{\beta_{1}} \epsilon \operatorname{csch}\left[\frac{1}{2} \sqrt{\beta_{1}}\left(l x+m y+\omega t+\xi_{0}\right)\right]^{2}}{\sqrt{\alpha \beta_{1}\left(\beta_{1} \gamma \omega^{2}-1\right)\left(\beta_{1} \gamma \omega^{2}+2\right)}\left(\epsilon \operatorname{coth}\left[\frac{1}{2} \sqrt{\beta_{1}}\left(l x+m y+\omega t+\xi_{0}\right)\right]+1\right)}\right)}{2 \sqrt{2}}
$$

$$
\begin{aligned}
U_{20}(x, y, t)= & \frac{1}{\sqrt{2}} \sqrt{1-\beta_{1}^{2} \gamma^{2} \omega^{4}}\left(\frac{1}{\sqrt{\alpha\left(\beta_{1} \gamma \omega^{2}-1\right)\left(\beta_{1} \gamma \omega^{2}+2\right)}}+\sqrt{\beta_{1}} \epsilon\left(\eta \cosh \left[\sqrt{\beta_{1}}\left(l x+m y+\omega t+\xi_{0}\right)\right]+1\right) /\right. \\
& \left(\eta+\cosh \left[\sqrt{\beta_{1}}\left(l x+m y+\omega t+\xi_{0}\right)\right]\left(\eta+\cosh \left[\sqrt{\beta_{1}}\left(l x+m y+\omega t+\xi_{0}\right)\right]\right.\right. \\
& \left.\left.\left.+\epsilon \sinh \left[\sqrt{\beta_{1}}\left(l x+m y+\omega t+\xi_{0}\right)\right]\right) \sqrt{\alpha \beta_{1}\left(\beta_{1} \gamma \omega^{2}-1\right)\left(\beta_{1} \gamma \omega^{2}+2\right)}\right)\right)
\end{aligned}
$$

$$
\begin{aligned}
U_{21}(x, y, t)= & \frac{1}{\sqrt{2}} \sqrt{1-\beta_{1}^{2} \gamma^{2} \omega^{4}}\left(\frac{1}{\sqrt{\alpha\left(\beta_{1} \gamma \omega^{2}-1\right)\left(\beta_{1} \gamma \omega^{2}+2\right)}}+\left(\epsilon \left(\eta \sqrt{p^{2}+1} \cosh \left[\sqrt{\beta_{1}}\left(l x+m y+\omega t+\xi_{0}\right)\right]+1\right.\right.\right. \\
& \left.\left.-p \sinh \left[\sqrt{\beta_{1}}\left(l x+m y+\omega t+\xi_{0}\right)\right]\right) \sqrt{\beta_{1}}\right) /\left(\cosh \left[\sqrt{\beta_{1}}\left(l x+m y+\omega t+\xi_{0}\right)\right]+\eta \sqrt{p^{2}+1}\right. \\
& \left(\eta \sqrt{p^{2}+1}+\cosh \left[\sqrt{\beta_{1}}\left(l x+m y+\omega t+\xi_{0}\right)\right]+\epsilon\left(\sinh \left[\sqrt{\beta_{1}}\left(l x+m y+\omega t+\xi_{0}\right)\right]+p\right)\right) \\
& \left.\left.\sqrt{\alpha \beta_{1}\left(\beta_{1} \gamma \omega^{2}-1\right)\left(\beta_{1} \gamma \omega^{2}+2\right)}\right)\right)
\end{aligned}
$$




\subsection{Simplified modified form of Camassa-Holm equation}

We consider a simplified modified form of $\mathrm{CH}$-equation as

$$
U_{t}+2 \beta U_{x}-U_{\mathrm{xxt}}+\delta U^{2} U_{x}=0,
$$

where $\beta$ and $\delta$ are non zero constants. Consider the wave transformation as; $U(x, y, t)=U(\xi), \xi=k x+\omega t$. By this transformation we obtained ordinary differential equation of Eq.(38) as

$$
\omega U^{\prime}+2 \beta k U^{\prime}-k^{2} \omega U^{\prime \prime \prime}+\delta k U^{2} U^{\prime}=0,
$$

we integrate Eq.(39) once time according to the $\xi$ and constant of integration equal to zero, then we obtained as

$$
\omega U+2 \beta k U-k^{2} \omega U^{\prime \prime}+\frac{1}{3} \delta k U^{3}=0 .
$$

We balance the nonlinear term and highest order derivative in Eq.(40) allow obtaining the value of $n=1$. The general solution of Eq.(40) takes the form of:

$$
U(\xi)=a_{0}+a_{1} \Psi(\xi)+\frac{b_{1}}{\Psi(\xi)}+d_{1} \frac{\Psi^{\prime}(\xi)}{\Psi(\xi)}
$$

Substituting Eq.(41) into Eq.(40) and combining each coefficients of $\Psi^{\prime} j(\xi) \Psi^{i}(\xi)(j=0,1 ; i=1,2,3, \ldots . n)$, then making a every coefficient equal to zero and obtaining a set of algebraic equations. We solve this system of equations with the aid of Mathematica. The parameters $a_{0}, a_{1}, b_{1}, d_{1}$ can be determined as

\section{Case-1}

$$
a_{0}=0, a_{1}= \pm \frac{\sqrt{6} \sqrt{\beta} \sqrt{\beta_{3}} k}{\sqrt{\beta_{1}(-\delta) k^{2}-2 \delta}}, b_{1}=0, d_{1}= \pm \frac{\sqrt{6} \sqrt{\beta} k}{\sqrt{\beta_{1}(-\delta) k^{2}-2 \delta}}, \omega=-\frac{4 \beta k}{\beta_{1} k^{2}+2} .
$$

Substituting the Eq.(42), only for the positive value of $a_{1}$ and $d_{1}$ in Eq.(41), the solutions of Eq.(38) are given as:

$$
\begin{aligned}
U_{1}(x, y, t)=- & \frac{\sqrt{\frac{3}{2}} \sqrt{\beta} k\left(2 \beta_{1} \sqrt{\beta_{3}}\left(\epsilon \operatorname{coth}\left[\frac{1}{2} \sqrt{\beta_{1}}\left(k x+\omega t+\xi_{0}\right)\right]+1\right)^{2}+\sqrt{\beta_{1}} \beta_{2} \epsilon \operatorname{csch}\left[\frac{1}{2} \sqrt{\beta_{1}}\left(k x+\omega t+\xi_{0}\right)\right]^{2}\right)}{\beta_{2} \sqrt{-\delta\left(\beta_{1} k^{2}+2\right)}\left(\epsilon \operatorname{coth}\left[\frac{1}{2} \sqrt{\beta_{1}}\left(k x+\omega t+\xi_{0}\right)\right]+1\right)} \\
U_{2}(x, y, t)= & \left(\sqrt { \frac { 3 } { 2 } } k \sqrt { \beta } \left(-2 \sqrt{\beta_{1}} \epsilon\left(\eta \cosh \left[\sqrt{\beta_{1}}\left(k x+\omega t+\xi_{0}\right)\right]+1\right)+\left(\eta+\cosh \left[\sqrt{\beta_{1}}\left(k x+\omega t+\xi_{0}\right)\right]\right.\right.\right. \\
& \left.\left.\left.+\epsilon \sinh \left[\sqrt{\beta_{1}}\left(k x+\omega t+\xi_{0}\right)\right]\right)^{2} \sqrt{\frac{\beta_{1}}{\beta_{3}}} \sqrt{\beta_{3}}\right)\right) /\left(\eta+\cosh \left[\sqrt{\beta_{1}}\left(k x+\omega t+\xi_{0}\right)\right]\right. \\
& \left.\left(\eta+\cosh \left[\sqrt{\beta_{1}}\left(k x+\omega t+\xi_{0}\right)\right]+\epsilon \sinh \left[\sqrt{\beta_{1}}\left(k x+\omega t+\xi_{0}\right)\right]\right) \sqrt{-\delta\left(\beta_{1} k^{2}+2\right)}\right)
\end{aligned}
$$




$$
\begin{aligned}
U_{3}(x, y, t)= & -\left(\sqrt { 6 } \sqrt { \beta } k \left(\left(\epsilon \left(\eta\left(-\sqrt{p^{2}+1}\right) \cosh \left[\sqrt{\beta_{1}}\left(k x+\omega t+\xi_{0}\right)\right]+p \sinh \left[\sqrt{\beta_{1}}\left(k x+\omega t+\xi_{0}\right)\right]-1 \sqrt{\beta_{1}}\right.\right.\right.\right. \\
& +\sinh \left[\sqrt{\beta_{1}}\left(k x+\omega t+\xi_{0}\right)\right]+p\left(2 \cosh \left[\sqrt{\beta_{1}}\left(k x+\omega t+\xi_{0}\right)\right]+2 \eta \sqrt{p^{2}+1}\right. \\
& \left.\left.+\epsilon\left(\sinh \left[\sqrt{\beta_{1}}\left(k x+\omega t+\xi_{0}\right)\right]+p\right)\right) \epsilon\left(\sinh \left[\sqrt{\beta_{1}}\left(k x+\omega t+\xi_{0}\right)\right]+p\right)\right) \\
& \sqrt{-\delta\left(\beta_{1} k^{2}+2\right)}\left(\frac{\epsilon\left(\sinh \left[\sqrt{\beta_{1}}\left(k x+\omega t+\xi_{0}\right)\right]+p\right)}{\cosh \left[\sqrt{\beta_{1}}\left(k x+\xi_{0}+t \omega\right)\right]+\eta \sqrt{p^{2}+1}}+1\right)
\end{aligned}
$$

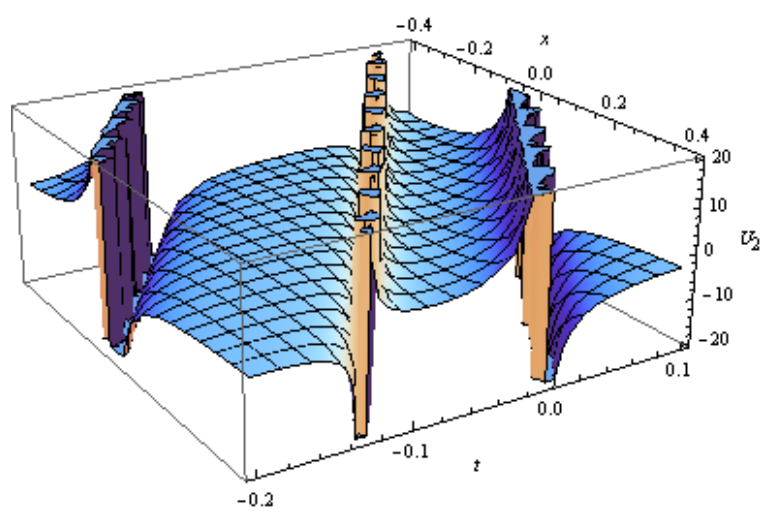

(a)

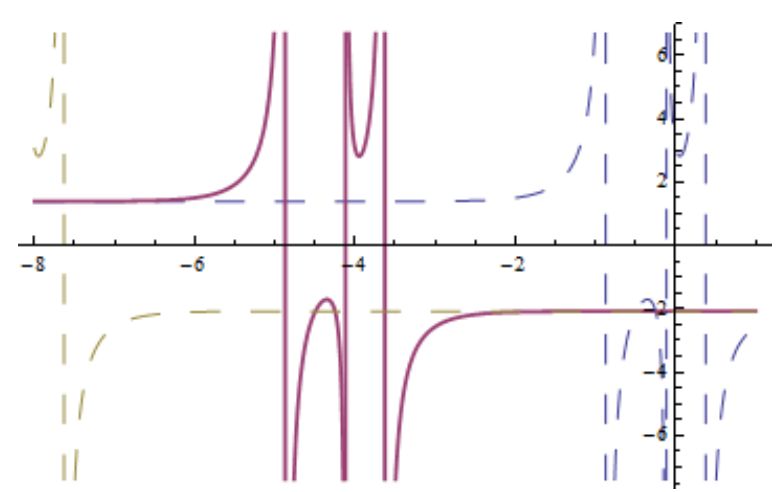

(b)

Figure 5: Solitary wave solution given in Eq.(44) when $\beta_{1}=2, \beta_{2}=4, \beta_{3}=2, \epsilon=5, \eta=-3, \xi_{0}=0.5, k=2, \omega=8, \alpha=0.2, \delta=-2$

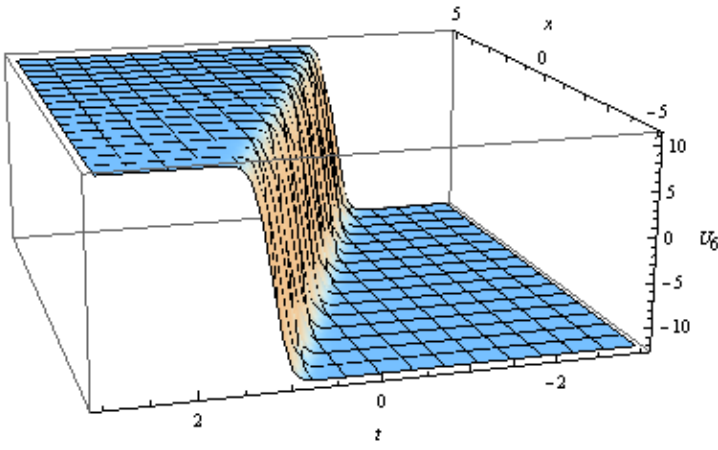

(a)

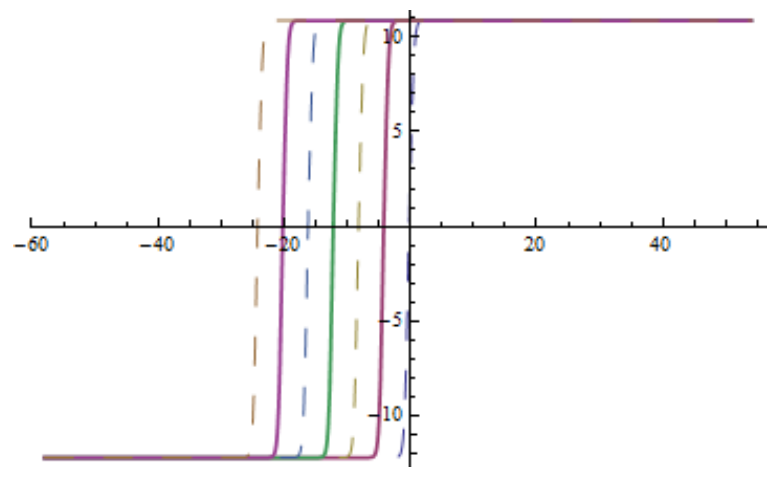

(b)

Figure 6: Solitary wave solution given in Eq.(49) when $\beta_{1}=4, \beta_{3}=2, \epsilon=-5, \eta=2, \xi_{0}=0.3, k=2, \omega=8, p=0.6, \alpha=0.2, \delta=-4$

\section{Case-II}

$$
\begin{gathered}
a_{0}= \pm \frac{\sqrt{6} \sqrt{\beta} \sqrt{\beta_{1}} k}{\sqrt{\beta_{1}(-\delta) k^{2}-2 \delta}}, a_{1}= \pm \frac{2 \sqrt{6} \sqrt{\beta} \sqrt{\beta_{3}} k}{\sqrt{\beta_{1}(-\delta) k^{2}-2 \delta}}, b_{1}=d_{1}=0, \\
\omega=-\frac{4 \beta k}{\beta_{1} k^{2}+2}, \beta_{2}= \pm 2 \sqrt{\beta_{1}} \sqrt{\beta_{3}} .
\end{gathered}
$$


Substituting Eq. (46), only the positive value of $a_{0}$ and $a_{1}$ into Eq. (41), the solutions of Eq. (38) can be given as:

$$
U_{4}(x, y, t)=-\frac{\sqrt{6} \sqrt{\beta} k\left(2 \beta_{1} \sqrt{\beta_{3}}\left(\epsilon \operatorname{coth}\left[\frac{1}{2} \sqrt{\beta_{1}}\left(k x+\omega t+\xi_{0}\right)\right]+1\right)-\sqrt{\beta_{1}} \beta_{2}\right)}{\beta_{2} \sqrt{-\delta\left(\beta_{1} k^{2}+2\right)}}
$$

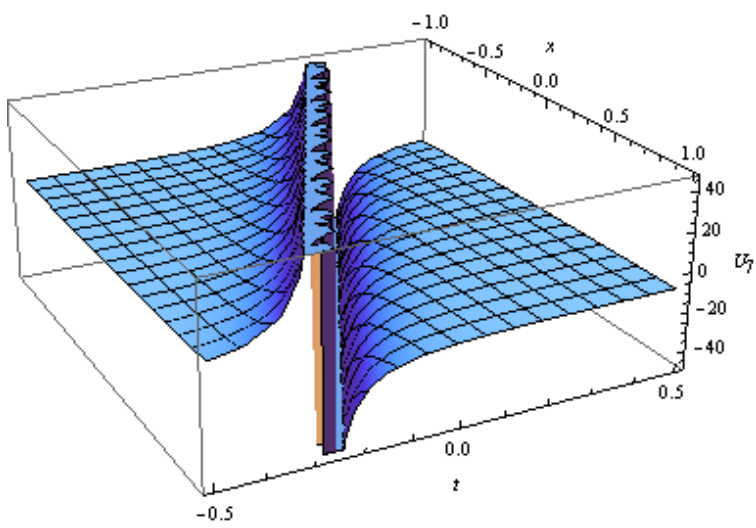

(a)

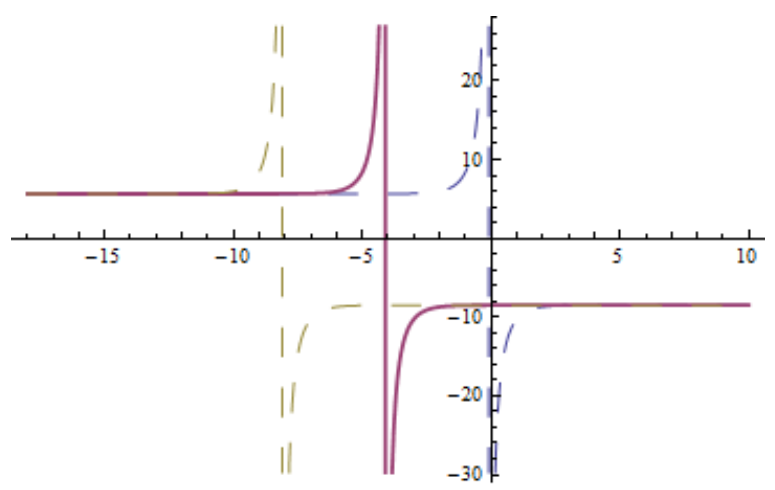

(b)

Figure 7: Solitary wave solution given in Eq. (51) when $\beta_{1}=1, \beta_{2}=2, \beta_{3}=1, \epsilon=5, \eta=3, \xi_{0}=0.2, k=2, \omega=8, \alpha=1, \delta=2$

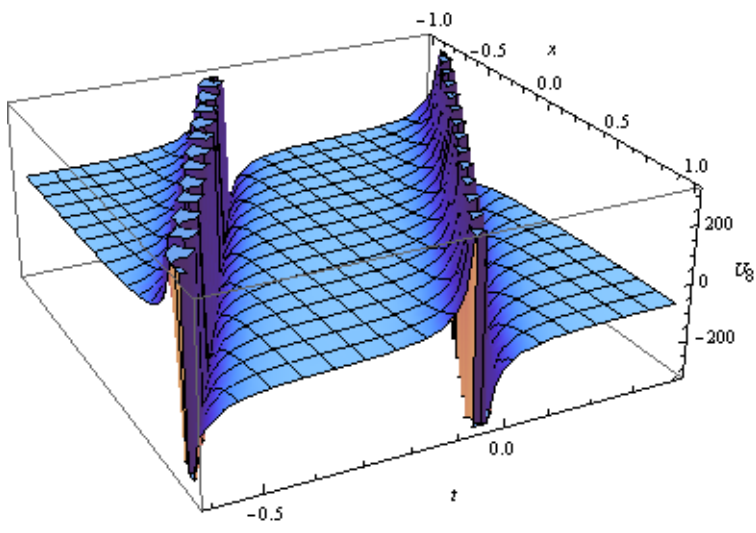

(a)

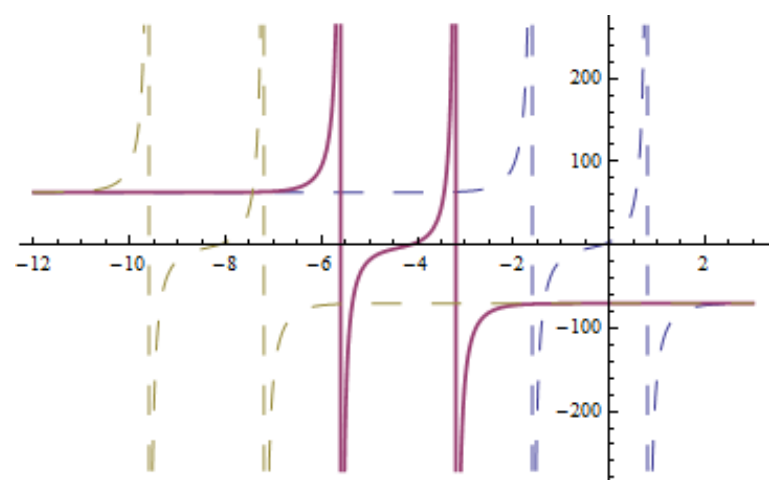

(b)

Figure 8: Solitary wave solution given in Eq. (52) when $\beta_{1}=2, \beta_{2}=4, \beta_{3}=2, \epsilon=18, \eta=-15, \xi_{0}=0.8, k=2, \omega=8, \alpha=8, \delta=2$

$$
\begin{aligned}
& U_{5}(x, y, t)=\left(\sqrt { 6 } k \sqrt { \beta } \left(\sqrt{\beta_{1}}\left(\eta+\cosh \left[\sqrt{\beta_{1}}\left(k x+\omega t+\xi_{0}\right)\right]\right)-\left(\eta+\cosh l e f t\left[\sqrt{\beta_{1}}\left(k x+\omega t+\xi_{0}\right)\right]\right.\right.\right. \\
&\left.+\epsilon \sinh \left[\sqrt{\beta_{1}}\left(k x+\omega t+\xi_{0}\right)\right]\right) \sqrt{\left.\left.\frac{\beta_{1}}{\beta_{3}} \sqrt{\beta_{3}}\right)\right) / \sqrt{-\delta\left(\beta_{1} k^{2}+2\right)}\left(\eta+\cosh \left[\sqrt{\beta_{1}}\left(k x+\omega t+\xi_{0}\right)\right]\right)} \\
& U_{6}(x, y, t)=\frac{\sqrt{6} \sqrt{\beta} k\left(\sqrt{\beta_{1}}+2 \sqrt{\beta_{3}}\left(-\frac{\epsilon\left(\sinh \left[\sqrt{\beta_{1}}\left(k x+\omega t+\xi_{0}\right)\right]+p\right)}{\cosh \left[\sqrt{\beta_{1}}\left(k x+\omega t+\xi_{0}\right)\right]+\eta \sqrt{p^{2}+1}}-1\right)\right)}{\sqrt{-\delta\left(\beta_{1} k^{2}+2\right)}}
\end{aligned}
$$




\section{Case-III}

$a_{0}=0, a_{1}= \pm \frac{2 \sqrt{3} \sqrt{\beta} \sqrt{\beta_{3}} k}{\sqrt{\beta_{1} \delta k^{2}-\delta}}, b_{1}=d_{1}=0, \omega=\frac{2 \beta k}{\beta_{1} k^{2}-1}$.

Substituting Eq.(50), only the positive value of $a_{1}$ into Eq.(41) the solutions of Eq.(38) can be given as:

$$
\begin{aligned}
& U_{7}(x, y, t)= \\
& -\frac{2 \sqrt{3} \sqrt{\beta} \beta_{1} \sqrt{\beta_{3}} k\left(\epsilon \operatorname{coth}\left[\frac{1}{2} \sqrt{\beta_{1}}\left(k x+\omega t+\xi_{0}\right)\right]+1\right)}{\beta_{2} \sqrt{\delta\left(\beta_{1} k^{2}-1\right)}}
\end{aligned}
$$

$$
\begin{aligned}
& U_{8}(x, y, t)= \\
& -\frac{\sqrt{3} \sqrt{\beta} \sqrt{\frac{\beta_{1}}{\beta_{3}}} \sqrt{\beta_{3}} k\left(\frac{\epsilon \sinh \left[\sqrt{\beta_{1}}\left(k x+\omega t+\xi_{0}\right)\right]}{\eta+\cosh \left[\sqrt{\beta_{1}}\left(k x+\omega t+\xi_{0}\right)\right.}+1\right)}{\sqrt{\delta\left(\beta_{1} k^{2}-1\right)}}
\end{aligned}
$$

$$
\begin{aligned}
& U_{9}(x, y, t)= \\
& \frac{2 \sqrt{3} \sqrt{\beta} \sqrt{\beta_{3}} k\left(-\frac{\epsilon\left(\sinh \left[\sqrt{\beta_{1}}\left(k x+\omega t+\xi_{0}\right)\right]+p\right)}{\cosh \left[\sqrt{\beta_{1}}\left(k x+\omega t+\xi_{0}\right)\right]+\eta \sqrt{p^{2}+1}}-1\right)}{\sqrt{\delta\left(\beta_{1} k^{2}-1\right)}}
\end{aligned}
$$

\section{Case-IV}

$a_{0}=a_{1}=0, b_{1}=0, d_{1}= \pm \frac{2 \sqrt{3} \sqrt{\beta} k}{\sqrt{-\delta-2 \beta_{1} \delta k^{2}}}, \omega=-\frac{2 \beta k}{2 \beta_{1} k^{2}+1}$

Substituting Eq. (54), only the positive value of $d_{1}$ into Eq. (41), the solutions of Eq. (38) can be given as:

$$
\begin{aligned}
& U_{10}(x, y, t)= \\
& -\frac{\sqrt{3} \sqrt{\beta} \sqrt{\beta_{1}} k \epsilon \operatorname{csch}\left[\frac{1}{2} \sqrt{\beta_{1}}\left(k x+\omega t+\xi_{0}\right)\right]^{2}}{\sqrt{-\delta\left(2 \beta_{1} k^{2}+1\right)}\left(\epsilon \operatorname{coth}\left[\frac{1}{2} \sqrt{\beta_{1}}\left(k x+\omega t+\xi_{0}\right)\right]+1\right)}
\end{aligned}
$$

$$
\begin{aligned}
& U_{11}(x, y, t)=2 \sqrt{3} \sqrt{\beta} \sqrt{\beta_{1}} k \epsilon\left(\eta \cosh \left[\sqrt{\beta_{1}}\left(k x+\omega t+\xi_{0}\right)\right]\right. \\
& +1) /\left(\eta+\cosh \left[\sqrt{\beta_{1}}\left(k x+\omega t+\xi_{0}\right)\right]\right. \\
& \sqrt{-\delta\left(2 \beta_{1} k^{2}+1\right)}\left(\eta+\cosh \left[\sqrt{\beta_{1}}\left(k x+\omega t+\xi_{0}\right)\right]\right. \\
& \left.\left.+\epsilon \sinh \left[\sqrt{\beta_{1}}\left(k x+\omega t+\xi_{0}\right)\right]\right)\right)
\end{aligned}
$$

$$
\begin{aligned}
& U_{12}(x, y, t)=2 \sqrt{3} \sqrt{\beta} \sqrt{\beta_{1}} k \epsilon\left(\eta \sqrt{p^{2}+1} \cosh \right. \\
& \left.\left[\sqrt{\beta_{1}}\left(k x+\omega t+\xi_{0}\right)\right]-p \sinh \left[\sqrt{\beta_{1}}\left(k x+\omega t+\xi_{0}\right)\right]+1\right) / \\
& \left(\cosh \left[\sqrt{\beta_{1}}\left(k x+\omega t+\xi_{0}\right)\right]\right. \\
& +\eta \sqrt{p^{2}+1}\left(\cosh \left[\sqrt{\beta_{1}}\left(k x+\omega t+\xi_{0}\right)\right]+\eta \sqrt{p^{2}+1}\right. \\
& \left.\left.+\epsilon\left(\sinh \left[\sqrt{\beta_{1}}\left(k x+\omega t+\xi_{0}\right)\right]+p\right)\right) \sqrt{-\delta\left(2 \beta_{1} k^{2}+1\right)}\right)
\end{aligned}
$$

\section{Case-V}

$$
\begin{aligned}
& a_{0}= \pm \frac{\sqrt{2} \sqrt{\beta}}{\sqrt{\delta}}, a_{1}= \pm \frac{2 \sqrt{2} \sqrt{\beta} \sqrt{\beta_{3}}}{\sqrt{\beta_{1}} \sqrt{\delta}}, b_{1}=d_{1}=0, k=\frac{1}{\sqrt{\beta_{1}}}, \\
& \omega=-\frac{4 \beta}{3 \sqrt{\beta_{1}}}, \beta_{2}= \pm 2 \sqrt{\beta_{1}} \sqrt{\beta_{3}}
\end{aligned}
$$

Substituting Eq. (58), only the positive value of $a_{0}$ and $a_{1}$ into Eq. (41), the solutions of Eq. (38) can be given as:

$$
\begin{aligned}
& U_{13}(x, y, t)= \\
& \frac{\sqrt{2} \sqrt{\beta}\left(\beta_{2}-2 \sqrt{\beta_{1}} \sqrt{\beta_{3}}\left(\epsilon \operatorname{coth}\left[\frac{1}{2} \sqrt{\beta_{1}}\left(k x+\omega t+\xi_{0}\right)\right]+1\right)\right)}{\beta_{2} \sqrt{\delta}} \\
& U_{14}(x, y, t)=\frac{\sqrt{2} \sqrt{\beta}\left(1-\frac{\sqrt{\frac{\beta_{1}}{\beta_{3}} \sqrt{\beta_{3}}\left(\frac{\epsilon \sinh \left[\sqrt{\beta_{1}}\left(k x+\omega t+\xi_{0}\right)\right]}{\eta+\cosh \left[\sqrt{\beta_{1}}\left(k x+\omega t+\xi_{0}\right)\right]}\right)}}{\sqrt{\beta_{1}}}\right)}{\sqrt{\delta}}
\end{aligned}
$$

$$
\begin{aligned}
& U_{15}(x, y, t)= \\
& \frac{\sqrt{2} \sqrt{\beta}\left(\frac{2 \sqrt{\beta_{3}}\left(-\frac{\epsilon\left(\sinh \left[\sqrt{\beta_{1}}\left(k x+\omega t+\xi_{0}\right)\right]+p\right)}{\cosh \left[\sqrt{\beta_{1}\left(k x+\omega t+\xi_{0}\right)}\right)+\eta \sqrt{p^{2}+1}}-1\right)}{\sqrt{\beta_{1}}}+1\right)}{\sqrt{\delta}}
\end{aligned}
$$

\section{Conclusion}

We have successfully applied a new method on two nonlinear evaluation equations. We have obtained a new exact traveling and solitary wave solutions of GZK-BBMequation and simplified modified form of $\mathrm{CH}$-equation by applying the Modified extended auxiliary equation mapping method. As a results, these new solutions are obtained in the form of elliptic functions, trigonometric functions, kink and antikink solitions, bright and dark solitons, periodic solitary wave and travelling wave solutions 
and also show two and three dimensional graphs with the help of Mathematica. These new families of solutions show the power, effectiveness, capability, realizabilities and fruitfulness of this new method. We can solve other nonlinear physical phenomena, which are related to nonlinear evaluation equations with the help of this new method.

\section{References}

[1] Wazwaz A.M., Compact and noncompact physical structures for the ZK-BBM equation, Appl. Math. Comput. 2005, 169, 713-725.

[2] Wazwaz A.M., The extended tanh method for new compact and noncompact solutions for the KP-BBM and the ZK-BBM equations, Chaos Solitons Fractals, 2008, 38, 1505-1516.

[3] Abdou M.A., Exact periodic wave solutions to some nonlinear evolution equations, Int. J. Nonlinear Sci., 2008, 6, 145-153.

[4] Mahmoudi J., Tolou N., Khatami I., Barari A., Ganji D.D., Explicit solution of nonlinear ZK-BBM wave equation using exp-function method, J. Appl. Sci., 2008, 8, 358-363.

[5] Wang Z.J., Tang S.Q., Bifurcation of travelling wave solutions for the generalized ZK-BBM equations, Commun. Nonlinear Sci. Numer. Simul., 2009, 14, 2948-2955.

[6] Song M., Yang C.X., Exact traveling wave solutions of the Zakharov-Kuznetsov-Benjamin-Bona-Mahony equation, Appl. Math. Comput., 2010, 216, 3234-3243.

[7] Camassa R., Holm D.D., An integrable shallow water equation with peaked Solitons, Physical Review Letters, 1993, 71, 16611664.

[8] Cooper F., Shepard H., Solitons in the Camassa-Holm shallow water equation, Physics Letters A, 1994, 194, 246-250.

[9] Liu Z.R., Qian. T.F., Peakons and their bifurcation in a generalized Camassa-Holm equation, International Journal of Bifurcation and Chaos, 2001, 11, 781-792.

[10] Zhang Z.D., Bi Q.S., Bifurcations of a generalized CamassaHolm equation, International Journal of Nonlinear Sciences and Numerical Simulation, 2005, 6, 81-86.

[11] Liu Z.R., Tang H., Explicit periodic wave solutions and their bifurcations for generalized Camassa-Holm equation, International Journal of Bifurcationl and Chaos, 2010, 20, 2507-2519.

[12] Deng S.F., Guo B.L., Wang T.C., Travelling wave solutions of a generalized Camassa-Holm-Degasperis-Procesi equation, Science China-Mathematics, 2011, 54, 555-572.

[13] Kalla C., Klein C. New construction of algebro-geometric solutions to the Camassa-Holm equation and their numerical evaluation, Proceedings of the Royal Society A- Mathematical Physical and Engineering Sciences, 2012, 468, 1371-1390.

[14] Song M., Liu Z., Periodic wave solutions and their limits for the ZK-BBM equation[J], Applied Mathematics \& Computation, 2012, 2012, 9-26.

[15] Adem K. R., Khalique C.M., Conservation Laws and Traveling Wave Solutions of a Generalized Nonlinear ZK-BBM Equation[J], Abstract \& Applied Analysis, 2014, 2014(2), 1-5.

[16] Roshid H.O., Roshid M.M., Rahman N., et al. New solitary wave in shallow water, plasma and ion acoustic plasma via the GZKBBM equation and the RLW equation[J], Propulsion \& Power Re- search, 2017, 6(1), 49-57.

[17] Khater A.H., Helal M.A., Seadawy A.R., General soliton solutions of $n$-dimensional nonlinear Schr?dinger equation, IL Nuovo Cimento, 2000, 115B, 1303-1312.

[18] Guo R., Hao H.Q., Zhang L.L., Dynamic behaviors of the breather solutions for the AB system in fluid mechanics, Nonlinear Dyn., 2013, 74, 701-709.

[19] Zhao X.J., Guo R., Hao H.Q., N-fold Darboux transformation and discrete soliton solutions for the discrete Hirota equation, Appl. Math. Lett., 2018, 75, 114-120.

[20] Yang Z.J., Zhang S.M., Li X.L., Pang Z.G., Variable sinh-Gaussian solitons in nonlocal nonlinear Schr?dinger equation, Appl. Math. Lett., 82, 64-70,(2018).

[21] Khater A.H., Callebaut D.K. and Seadawy A.R., General soliton solutions of an n-dimensional Complex Ginzburg-Landau equation, Physica Scripta, 2000, 62, 353-357.

[22] Seadawy A.R., Stability analysis of traveling wave solutions for generalized coupled nonlinear KdV equations, Appl. Math. Inf. Sci., 2016, 10, 1, 209-214.

[23] Khater A.H., Callebaut D.K., Helal M.A. and Seadawy A.R., Variational Method for the Nonlinear Dynamics of an Elliptic Magnetic Stagnation Line, The European Physical Journal D, 2006, 39, 237-245.

[24] Seadawy A.R., The generalized nonlinear higher order of KdV equations from the higher order nonlinear Schrodinger equation and its solutions, Optik - International Journal for Light and Electron Optics, 2017, 139, 31-43.

[25] Seadawy A.R. Ion acoustic solitary wave solutions of twodimensional nonlinear Kadomtsev-Petviashvili-Burgers equation in quantum plasma, Mathematical methods and applied Sciences, 2017, 40, (5), 1598-1607.

[26] Lenells. Traveling wave solutions of the Camassa-Holm equation[J]. Journal of Differential Equations, 2005, 217(2), 393-430.

[27] Kalisch H., Lenells J., Numerical study of traveling-wave solutions for the Camassa-Holm equation[J], Chaos Solitons \& Fractals, 2005, 25(2), 287-298.

[28] Baleanu1 D., Inc M., Yusuf A., Aliyu I.A., Traveling wave solutions and conservation laws for nonlinear evolution equation, Journal of Mathematical Physics, 2018, 59, 023506.

[29] Baleanu1 D., Inc M., Yusuf A., Aliyu I.A., Optical solitons, nonlinear self-adjointness and conservation laws for Kundu-Eckhaus equation, Chinese Journal of Physics, 2017, 55, 2341-2355.

[30] Inc M., Yusuf A., Aliyu I.A., Dark optical and other soliton solutions for the three different nonlinear Schr?dinger equations, Opt Quant Electron 2017, 49, 354.

[31] Inc M., Yusuf A., Aliyu I.A., Baleanu D., Soliton solutions and stability analysis for some conformable nonlinear partial differential equations in mathematical physics, Opt Quant Electron, 2018, 50, 190.

[32] Inc M., Yusuf A., Aliyu I.A., Baleanu D., Soliton structures to some time-fractional nonlinear differential equations with conformable derivative, Opt Quant Electron, 2018, 50, 20.

[33] Inc M., Yusuf A., Aliyu I.A., Baleanu D., Dark and singular optical solitons for the conformable space-time nonlinear Schr?dinger equation with Kerr and power law nonlinearity, Optik, 2018, 162, 65-75.

[34] Lu D., Seadawy A.R., Ali A., Dispersive traveling wave solutions of the Equal-Width and Modified Equal-Width equations via mathematical methods and its applications, Results in Physics, $2018,9$. 
[35] Helal M.A. and Seadawy A.R., Exact soliton solutions of an Ddimensional nonlinear Schrödinger equation with damping and diffusive terms, Z. Angew. Math. Phys. (ZAMP) 2011, 62, 839847.

[36] Khater A.H., Callebaut D.K., Malfliet W. and Seadawy A.R., Nonlinear Dispersive Rayleigh-Taylor Instabilities in Magnetohydrodynamic Flows, Physica Scripta, 2001, 64, 533-547.

[37] Lu D., Seadawy A.R., Khater M.A., Structure of solitary wave solutions of the nonlinear complex fractional generalized Zakharov dynamical system, Advances in Difference Equations, 2018, 2018, (1), 266.

[38] Ali A., Seadawy A.R., Lu D., New solitary wave solutions of some nonlinear models and their applications, Advances in Difference Equations, 2018, 2018,(1), 232.

[39] Seadawy A.R., Travelling wave solutions of a weakly nonlinear two-dimensional higher order Kadomtsev-Petviashvili dynamical equation for dispersive shallow water waves, The European Physical Journal Plus, 2017, 132, 29, 1-13.

[40] Seadawy A.R., Fractional travelling wave solutions of the higher order extended $\mathrm{KdV}$ equations in a stratified shear flow, part I, Computers and Mathematics with Applications, 2015, 70, 345352.

[41] Lu D., Seadawy A.R., Arshad M., Bright-dark solitary wave and elliptic function solutions of unstable nonlinear Schrödinger equation and their applications[]]. Optical \& Quantum Electronics, 2018, 50(1):23.

[42] Iqbal M., Seadawy A.R. and Lu D., Construction of solitary wave solutions to the nonlinear modified Kortewege-de Vries dynamical equation in unmagnetized plasma via mathematical methods, Modern Physics Letters A, 2018, 33, 1850183, 1-13.

[43] Arshad M., Seadawy A.R., Lu D., Exact bright-dark solitary wave solutions of the higher-order cubic-quintic nonlinear SchrOdinger equation and its stability, Optik 2017, 138, 40-49.
[44] Lu D., Seadawy A.R., Arshad M., Wang J., New solitary wave solutions of $(3+1)$-dimensional nonlinear extended ZakharovKuznetsov and modified KdV-Zakharov-Kuznetsov equations and their applications, Results Phys, 2017, 7, 899-909.

[45] Tariq K., Seadawy A.R., Bistable Bright-Dark solitary wave solutions of the $(3+1)$-dimensional Breaking soliton, Boussinesq equation with dual dispersion and modified Kortewegde Vries Kadomtsev Petviashvili equations and their applications, Results Phys 2017, 7, 1143-1149.

[46] Seadawy A.R., Traveling wave solutions of the Boussinesq and generalized fifth-order KdV equations by using the direct algebraic method, Appl Math Sci 2012, 6, (82), 4081-4090.

[47] Seadawy A.R., Stability analysis solutions for nonlinear threedimensional modified Korteweg-de Vries Zakharov Kuznetsov equation in a magnetized electron-positron plasma, Phys $\mathrm{A}$ 2016, 455, 44.

[48] Seadawy A.R., Approximation solutions of derivative nonlinear Schrodinger equation with computational applications by variational method, The European Physical Journal Plus, 2015, 130, 182, 1-10.

[49] Iqbal M., Seadawy A.R. and Lu D., Dispersive solitary wave solutions of nonlinear further modified Kortewege-de Vries dynamical equation in a unmagnetized dusty plasma via mathematical methods, Modern Physics Letters A, 2018, 33, 1850217, 1-19.

[50] Seadawy A.R., Manafian J., New soliton solution to the longitudinal wave equation in a magneto-electro-elastic circular rod, Results in Physics, 2018, 8, 1158-1167.

[51] Lu D., Seadawy A.R., Iqbal M., Mathematical physics via construction of traveling and solitary wave solutions of three coupled system of nonlinear partial differential equations and their applications, Results in Physics, 2018, 11, 1161-1171. 